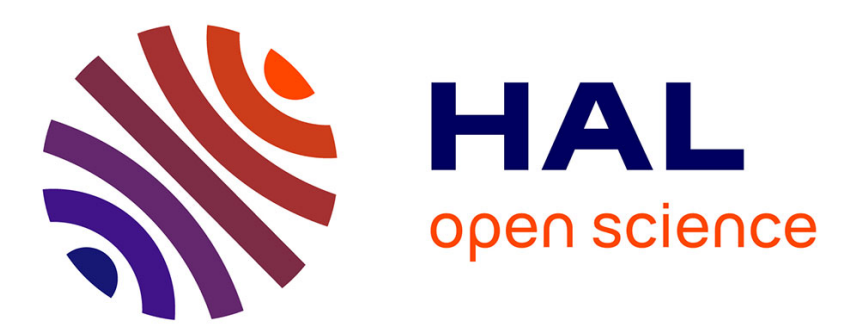

\title{
Application des caractéristiques géoacoustiques d'une argile artificielle à l'étude du champ acoustique d'une antenne multi-éléments
}

J. Bresson, J.-P. Longuemard

\section{- To cite this version:}

J. Bresson, J.-P. Longuemard. Application des caractéristiques géoacoustiques d'une argile artificielle à l'étude du champ acoustique d'une antenne multi-éléments. Revue de Physique Appliquée, 1985, 20 (6), pp.409-414. 10.1051/rphysap:01985002006040900 . jpa-00245352

\section{HAL Id: jpa-00245352 https://hal.science/jpa-00245352}

Submitted on 1 Jan 1985

HAL is a multi-disciplinary open access archive for the deposit and dissemination of scientific research documents, whether they are published or not. The documents may come from teaching and research institutions in France or abroad, or from public or private research centers.
L'archive ouverte pluridisciplinaire HAL, est destinée au dépôt et à la diffusion de documents scientifiques de niveau recherche, publiés ou non, émanant des établissements d'enseignement et de recherche français ou étrangers, des laboratoires publics ou privés. 


\title{
Application des caractéristiques géoacoustiques d'une argile artificielle à l'étude du champ acoustique d'une antenne multi-éléments
}

\author{
J. Bresson et J.-P. Longuemard (*) \\ Laboratoire de Mécanique, d'Acoustique et d'Instrumentation, \\ Université de Perpignan, Avenue de Villeneuve, 66025 Perpignan, France \\ (Reçu le 4 octobre 1984, révisé le 17 décembre 1984, accepté le 12 mars 1985)
}

\begin{abstract}
Résumé. - La détection d'objets enfouis dans les sédiments marins ou la détermination de certaines de leurs caractéristiques géotechniques peut se faire à partir d'ondes acoustiques générées et captées par des antennes multiéléments. Les auteurs se proposent d'appliquer des données, acquises en mer et à partir de sédiments artificiels, à un modèle numérique permettant de prévoir le comportement de la focalisation d'ondes en fonction de paramètres géoacoustiques des fonds marins.
\end{abstract}

L'influence du sol sera prise en compte :

- au niveau de l'interface,

- dans le sédiment.

Les paramètres acoustiques retenus dans le modèle (célérité et atténuation des ondes de compression - impédance acoustique des sédiments) influent sur la forme et les dimensions de la tache focale ainsi que sur les limites d'utilisation de ce procédé. Une trop forte atténuation des ondes interdit d'une part une bonne concentration de l'énergie acoustique à l'émission, d'autre part gêne le traitement du signal en réception (réduction trop importante des niveaux d'énergie des trajets les plus longs).

Les auteurs présentent également des résultats obtenus sur un sédiment artificiel permettant :

- d'une part de quantifier les paramètres acoustiques d'un site d'essais représentatif du comportement en milieu marin,

- d'autre part de définir les limites d'utilisation d'une antenne multi-éléments basse fréquence.

\begin{abstract}
The acoustic waves produced and received by a multi array transducer allows the detection of buried targets in marine sediments or the determination of their geoacoustic parameters.

In this study sea data obtained in situ and artificial sediment are used to modelise the focalization acoustic wave.

The characteristics of the sediment and the interface taken into account for a model.

These acoustic parameters (celerity and attenuation of the longitudinal waves - acoustic impedance of sediments) affect the form, the dimensions of the focal point and the performances of the process. A large wave attenuation enables the emission of energy focalization and troubles the treatment of the signal of reception (the energy level of the longer distances are too low).

In the paper results from artificial sediments are also presented. They are used to quantify acoustic parameters relative to an artificial site representing the natural behaviour of the sea. Furthermore these parameters define the performances of array transducers.
\end{abstract}

\section{Introduction.}

La connaissance des conditions de focalisation des ondes acoustiques dans les milieux stratifiés présente plusieurs intérêts (reconnaissance de certaines qualités mécaniques ou géotechniques des couches stratifiées,

(*) A compter du 1-1-85: Ecole Centrale des Arts et Manufactures, Grande Voie des Vignes, 92290 Chatenay Malabry. recherche d'objets enfouis). Dans tous les cas, la connaissance du comportement des ondes intervient d'une part aux interfaces, d'autre part durant le parcours dans les diverses couches. Les paramètres géotechniques et acoustiques qui nous ont paru les plus intéressants dans le cadre de cette étude sont les suivants :

- la masse volumique $(\rho)$ qui découle de la porosité ou de la teneur en eau des sédiments saturés; 
- la célérité $(c)$ et l'atténuation $(\delta)$ des ondes de compression.

Ces derniers paramètres sont du reste fonction de la masse volumique et de la minéralogie des sédiments [1-2-3]. Par voie de conséquence, l'impédance acoustique sera fonction de $\rho(Z s=\rho c)$. Ce facteur aura deux rôles :

- calcul de l'angle réfracté et de l'énergie entrant dans le sédiment;

- définition de l'atténuation soit directement, soit à travers la porosité.

\section{Etude théorique.}

2.1 RAPPEL SUR LA THÉORIE DE LA FOCALISATION BF. - Trois cas sont à considérer :

— focalisation à l'émission,

- focalisation utilisée en réception,

- combinaison des deux cas précédents.

Dans le premier cas, la focalisation est donnée par la somme de signaux acoustiques provenant de $p$ sources situées sur $n$ couronnes. Pour un milieu homogène non atténuant, la pression présente en un point est définie par :

(pour une couronne)

$$
\underset{\text { partiel }}{P(\theta)}=\sum_{j=1}^{p} \frac{A}{r_{j}} \cos \left(\omega t-k r_{j}\right)
$$

(pour n couronnes)

$$
\underset{\text { total }}{P(\theta)}=\sum_{i=1}^{n} \sum_{j=1}^{p} \frac{A}{r_{i j}} \cos \left(\omega t-k r_{i j}\right)
$$

où :

$r_{i j} \simeq D-a_{i} \Delta_{j}\left(\Delta_{j}\right.$ étant le cosinus directeur) est la distance séparant le point source d'un transducteur élémentaire.

$A$ est l'amplitude de la pression émise par un transducteur élémentaire.

$D$ est la distance séparant le point source du centre de l'antenne.

Or, pour obtenir l'énergie maximale qui définit le point de focalisation, les pressions acoustiques partielles $\frac{A}{r_{i j}}\left[\cos \left(\omega t-k r_{i j}\right)\right]$ doivent être en phase. Deux solutions peuvent permettre de respecter ce critère :

- placer les éléments émetteurs sur une calotte sphérique,

- placer ces mêmes éléments sur une embase plane circulaire et réaliser une commande de déphasage par moyens électroniques.

Ce dernier procédé permettra également de compenser les aberrations dues à la réfraction des ondes.

Dans le cas d'une focalisation à la réception, nous n'utiliserons plus la sommation des pressions acoustiques délivrées par chaque capteur, mais le produit des pressions sommées correspondant à chaque couronne $[10,11]$.

Si l'on définit par $F_{i}\left(a_{i}, \theta\right)$ le niveau fournit par une couronne le signal résultant vaudra :

soit

$$
\begin{aligned}
& G(\theta)=\prod_{i=1}^{n} F_{i}\left(a_{i}, \theta\right) \\
& G(\theta)=\prod_{i=1}^{n} \sum_{j=1}^{p} A_{i j}\left(a_{i}, \theta\right)
\end{aligned}
$$

où $A_{i j}$ est la pression reçue par le capteur $j$ de la couronne $i$.

Dans le cas des trois couronnes

$$
P \text { final }=G(\theta)=\frac{1}{4}\left[A_{1} A_{2} A_{3} \cos \left(3 \omega t+\alpha_{1}+\alpha_{2}+\alpha_{3}\right)+X A_{1} A_{2} A_{3} \cos (\omega t+\alpha)\right]
$$

où :

$$
\alpha=\operatorname{arctg} \frac{X_{2}}{X_{1}}
$$

et

$$
X=\sqrt{X_{1}^{2}+X_{2}^{2}}
$$

avec :

$$
X_{1}=\cos \left(\alpha_{1}+\alpha_{2}-\alpha_{3}\right)+\cos \left(\alpha_{2}+\alpha_{3}-\alpha_{1}\right)+\cos \left(\alpha_{3}+\alpha_{1}-\alpha_{2}\right)
$$

et

$$
X_{2}=\sin \left(\alpha_{1}+\alpha_{2}-\alpha_{3}\right)+\sin \left(\alpha_{2}+\alpha_{3}-\alpha_{1}\right)+\sin \left(\alpha_{3}+\alpha_{1}-\alpha_{2}\right) .
$$

Les facteurs $A_{i}$ et $\alpha_{i}$ correspondent, pour des trajets directs sans atténuation dans le cas des couronnes concentriques, à une pression acoustique $A_{i}$ et un déphasage $\alpha_{i}$. 


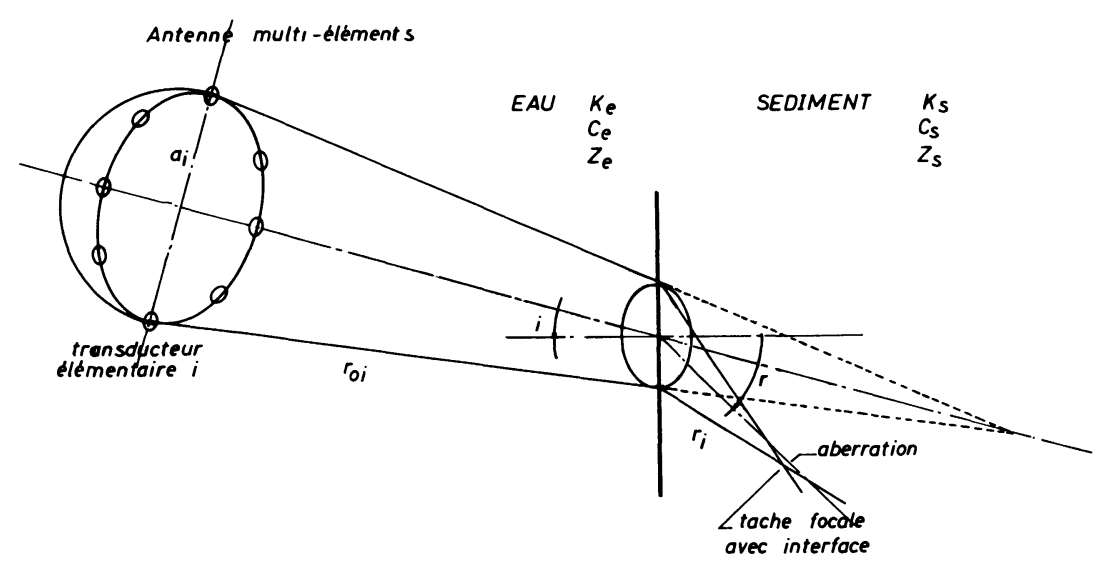

Fig. 1. - Schéma des trajets acoustiques avec et sans interface.

[Acoustics beam with and without substract.]

2.2 INFLUENCE DES QUALITÉS GÉOACOUSTIQUES DU SÉDIMENT (OU MILIEU POREUX QUELCONQUE).

2.2.1 Influence de la célérité. - La discontinuité à l'interface eau-sédiment provoque deux phénomènes $[6,3,8]$,

- le dépointage de l'antenne à cause de la loi de Snell Descartes (Fig. 1),

- des aberrations pour le faisceau focalisé [5].

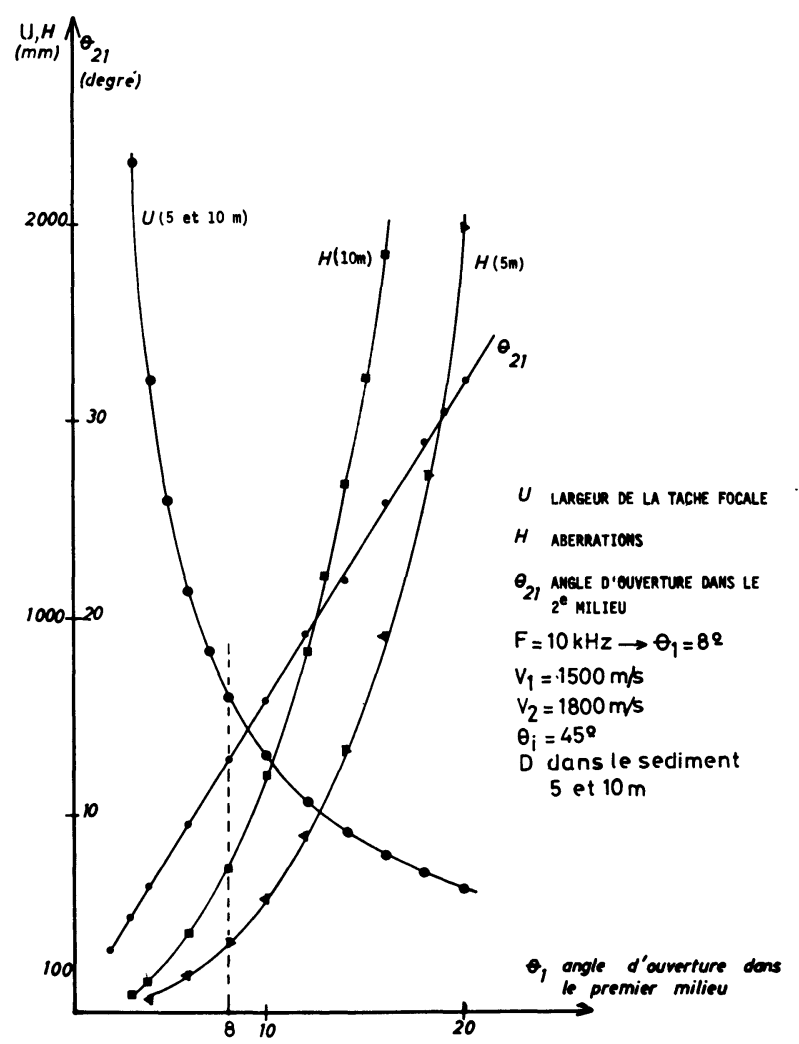

Fig. 2. - Evolution des aberrations, de la largeur de la tache focale et de l'angle d'ouverture dans le $2^{\mathrm{e}}$ milieu en fonction de l'angle d'ouverture dans le $1^{\mathrm{er}}$ milieu.

[Aberrations, width of focusing spot and aperture angle in the second element versus the aperture angle in the first element.]

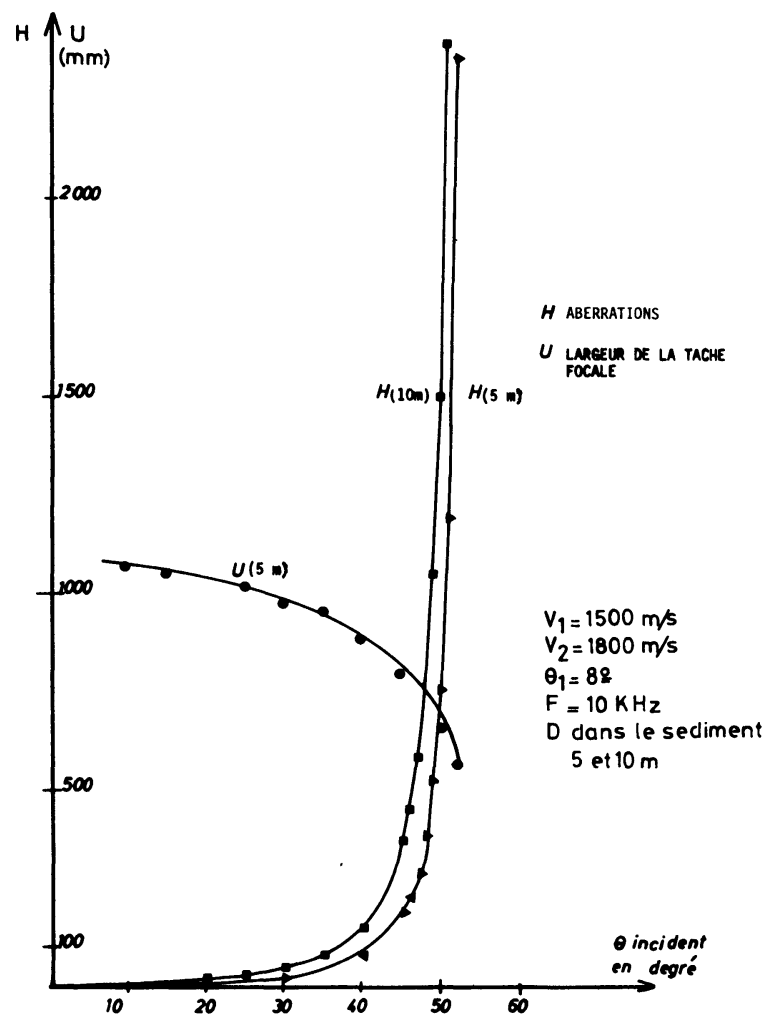

Fig. 3. - Evolution des aberrations et de la largeur de la tache focale en fonction de l'angle d'incidence de l'antenne multi-éléments.

[Evolution of aberrations and width of focusing spot versus the incidence angle of the array transduceurs.]

Le dépointage occasionné par la discontinuité d'impédance engendre un écart entre le foyer théorique et le foyer réel d'autant plus important que la célérité des ondes dans le sédiment est grande (Figs 2 et 3 ). Ces résultats ont été obtenus par calculs basés sur des données de propagations acoustiques acquises en nature et en laboratoire. Les échantillons analysés, de différentes provenances (carottes de grands fonds, plateaux continentaux côtiers) sont représentatifs 
du comportement acoustique des sédiments de surface [9] dont la masse volumique est comprise entre $1,45 \times 10^{3}$ et $2,1 \times 10^{3} \cdot \mathrm{kg} \cdot \mathrm{m}^{-3}$. Deux phénomènes physiques sont à prendre en considération :

- l'évolution de l'angle réfracté. Or, en fonction de l'angle incident $\theta_{i}$

$$
\theta_{\mathrm{r}}=\arcsin \left(\frac{c_{\mathrm{s}}}{c_{\mathrm{e}}} \sin \theta_{\mathrm{i}}\right)
$$

où :

$c_{s}$ et $c_{e}$ sont les célérités dans le sol et dans l'eau

- le niveau de l'onde transmise dans le sol $T$ vaut :

$$
\begin{aligned}
& T=1-R \\
& T=1-\left(\frac{Z_{\mathrm{e}} \cos \theta_{\mathrm{r}}-Z_{\mathrm{s}} \cos \theta_{\mathrm{i}}}{Z_{\mathrm{e}} \cos \theta_{\mathrm{r}}+Z_{\mathrm{s}} \cos \theta_{\mathrm{i}}}\right)^{2}
\end{aligned}
$$

où :

$Z_{\mathrm{e}}$ et $Z_{\mathrm{s}}$ sont les impédances de l'eau et du sédiment.

L'influence du sédiment a deux rôles qui peuvent être définis en fonction de sa masse volumique $[4,9$, $12,13]$.

$$
c_{\mathrm{s}}=a+b(1-\rho)^{n}
$$

$a$ et $b$ sont des constantes fonction de la minéralogie et $\rho$ est exprimée en $\mathrm{t} / \mathrm{m}^{3}$.

Cette modélisation convient aux sédiments de surface dans lesquels des ondes de fréquences comprises entre 10 et $30 \mathrm{kHz}$ sont susceptibles de se propager sur quelques mètres. Les facteurs $a$ et $b$ sont dépendants de la teneur en carbonate $\psi$ en $\%$. Un sable de Mer d'Iroise permettra, à masse volumique identique, une célérité plus grande que celui du Golfe du Lion :

$$
\begin{aligned}
& a_{\mathrm{ms}^{-1}}=1408+0,8 \psi \\
& b_{\mathrm{ms}^{-1}}=600+4 \psi .
\end{aligned}
$$

L'impédance du fond marin $Z_{\mathrm{s}}$ aura pour expression :

$$
Z_{\mathrm{s}}=\rho\left[a+b(1-\rho)^{n}\right] .
$$

Si l'on considère que l'impédance de l'eau de mer est constante et a une valeur proche de $1,6 \times 10^{6} \mathrm{~kg} \mathrm{~m}^{-2} \mathrm{~s}^{-1}$, la masse volumique de l'argile ou du deuxième milieu aura une influence prépondérante; en effet un sable permettant une célérité de $1800 \mathrm{~ms}^{-1}$ engendre une valeur d'angle critique de l'ordre de 45 à $50^{\circ}$. La réflexion des ondes étant totale à partir de cette valeur, il ne sera plus possible de focaliser. Nous utiliserons donc un modèle ne faisant pas intervenir de fort angle d'incidence, ce qui va du reste dans le sens pratique du but recherché : réduire les pertes au minimum.

2.2.2 Influence de l'atténuation. - De nombreux auteurs ont étudié expérimentalement l'atténuation représentée par un décrément $(\delta)$ dans les sédiments [7]. Plusieurs formes de relations existent et prennent en considération la croissance de $\delta$ avec la fréquence. La représentation globale serait la suivante [1].

$$
\delta=a+b F^{n}
$$

Nous considérons un modèle faisant intervenir une croissance linéaire de $\delta$ avec la fréquence [2].

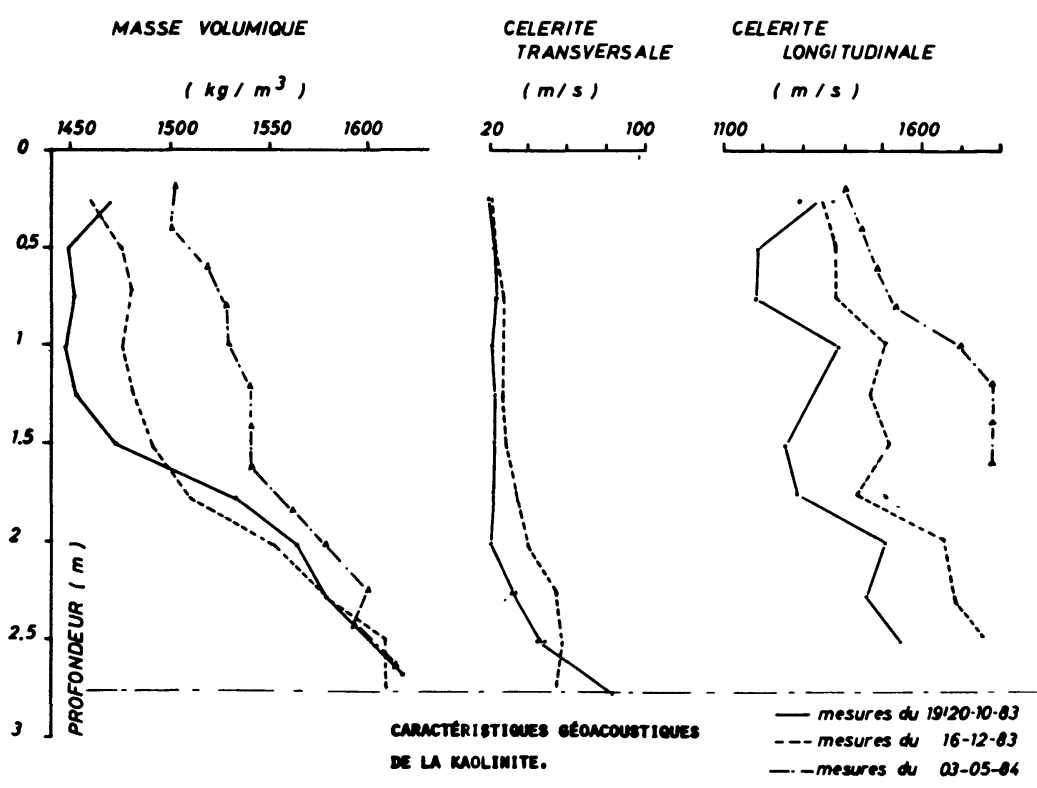

Fig. 4. - Paramètres géoacoustiques d'un site d'essais.

[in situ geoacoustical parameters.] 
2.2.3 Définition d'un site d'essais. - L'introduction de ce facteur dans l'étude de la focalisation pourra se faire à travers $A_{i j}$ suivant le modèle ci-dessous qui prend en compte :

$$
\begin{aligned}
& P=P_{0} \exp (-\xi r), \text { pour une onde plane } \\
& P=\frac{P_{0}}{r} \exp (-\xi r), \text { pour une onde sphérique }
\end{aligned}
$$

où :

$\xi_{\mathrm{np} / \mathrm{m}}=\frac{\delta \mathrm{d} B / m}{8,68}$ est le décrément d'atténuation en pression.

Alors que les corrélations concernant la célérité des ondes se présentent sous un aspect simple, l'étude de $\delta$ semble plus complexe et davantage liée aux sites expérimentaux. Nous avons, pour un matériau expérimental connu, constitué un bassin rempli d'argile artificielle dont les caractéristiques géoacoustiques sont les suivantes (Fig. 4) :

- croissance de la masse volumique avec la profondeur de l'argile dans la fosse $\left(1,5 \mathrm{t} . \mathrm{m}^{-3}<\rho<\right.$ $1,7 \mathrm{t} . \mathrm{m}^{-3}$ ),

- célérité à l'interface $c_{\mathrm{s}}$ de $1520 \mathrm{~ms}^{-1}$,

- célérité à $2 \mathrm{~m}$ de profondeur $1640 \mathrm{~ms}^{-1}$,

- atténuation variant de 0,6 à plusieurs $\mathrm{dB}$ par longueur d'onde.

Les essais de focalisation pourront se faire dans une gamme de fréquence comprise entre quelques $\mathrm{kHz}$ et $50 \mathrm{kHz}$.

La valeur de la célérité en surface de l'argile, liée à une forte teneur en eau, présente deux avantages :

- une grande quantité d'énergie transmise à l'interface,

- valeur éloignée de l'angle critique.

En effet, la valeur moyenne de $R$, mesurée au réflectomètre entre 10 et $150 \mathrm{kHz}$, en général est de $-17 \mathrm{~dB}$, ce qui assure une valeur de $T$ peu différente de 1 pour les angles incidents utilisés (maximum de $30^{\circ}$ ).

3. Introduction des facteurs géoacoustiques dans les modèles de base.

3.1 CAS DE L'ÉMISSION. - Les transducteurs sont placés par huit sur quatre couronnes concentriques.

La symétrie du système permet de simplifier l'écriture. La pression au point de focalisation provoquée par la couronne $i$, dans le cas particulier où cette dernière est perpendiculaire à l'axe de propagation, sera définie par l'équation ci-dessous :

$$
\begin{aligned}
P_{i}=\frac{P_{0} \exp -\xi r_{i}}{r_{0 i}+r_{i}} & (1-R)^{1 / 2} \times \\
& \times \exp j\left(\omega t-K_{\mathrm{e}} r_{0 i}-K_{\mathrm{s}} r_{i}\right)
\end{aligned}
$$

où :

$K_{\mathrm{e}}$ et $K_{\mathrm{s}}$ sont les nombres d'ondes dans l'eau et le sédiment,

$r_{0 i}$ et $r_{i}$ sont les longueurs des trajets acoustiques dans l'eau et le sédiment pour la couronne $i$.

L'énergie due à l'ensemble des couronnes $I_{\text {result }}$ si l'on pose :

$$
P_{i}=P_{0 i}^{\prime} \exp j\left(\omega t-\alpha_{i}\right),
$$

vaut

$$
I_{\text {result }} \simeq \sum_{i}^{n}\left(P_{i}\right)^{2}
$$

3.2 CAS DE LA RÉCEPTION. - Il s'agit, cette fois, de faire intervenir $R$ et $\delta \operatorname{sur} A_{1}$, puis $A_{2}$ et enfin $A_{3}$, en effet une quatrième couronne n'améliorerait pas les résultats. Après filtrage, de manière à ne conserver que le terme en $\cos \omega t$, la configuration géométrique fournira un produit de la forme :

$$
\begin{aligned}
G(\theta)=\frac{K P_{0}^{3}}{} & \times\left(1-R_{1}\right)^{1 / 2}\left(1-R_{2}\right)^{1 / 2}\left(1-R_{3}\right)^{1 / 2} \\
& \left(r_{01}+r_{1}\right)\left(r_{02}+r_{2}\right)\left(r_{03}+r_{3}\right) \\
& \times \exp -\xi\left(r_{1}+r_{2}+r_{3}\right) \cos (\omega t-\alpha)
\end{aligned}
$$

3.3 Application ET DisCUSSION. - Les coefficients de réflexion $R_{i}$ varient de 0,08 pour une argile sous incidence nulle à 1 pour un sable et une incidence proche de $45^{\circ}$ alors que $\delta$ sera considéré comme linéaire entre quelques kilohertz et quelques dizaines de kilohertz [7, 8].

Les faisceaux émis par la couronne externe en émission ou captée par cette dernière en réception ont deux motifs pour n'avoir qu'une faible influence par rapport au faisceau central : augmentation de $R_{i}$ et de $r_{i}$. La longueur de $r_{i}$ intervient deux fois directement (trajet aller-retour).

Alors que $\boldsymbol{R}_{i}$ a une influence simple en émission, dans le cas de la réception le traitement en produit entraîne une altération de la qualité de la focalisation. Un remède pourrait toutefois être apporté par une commande de gain électronique permettant de s'affranchir des effets dus à l'interface. Dans ce cas le calcul numérique, basé sur les équations 1 et 2 montre que la focalisation est impossible lorsque $\delta$ est supérieur à $10 \mathrm{~dB} \mathrm{~m}^{-1} \mathrm{kHz}^{-1}$.

Une application est donnée pour les valeurs suivantes (Fig. 5) :

$$
\begin{aligned}
& R_{0}=1 \mathrm{~m} \\
& f=30 \mathrm{kHz} \\
& 0,5 \mathrm{~dB} \mathrm{~m}^{-1} \mathrm{kHz}^{-1}<\delta<5 \mathrm{~dB} \mathrm{~m}^{-1} \mathrm{kHz}^{-1} \\
& \rho=1,5 \mathrm{t} \cdot \mathrm{m}^{-3} \\
& C_{\mathrm{s}} \text { argile }=1520 \mathrm{~ms}^{-1} \\
& Z_{\mathrm{s}} \text { argile }>2,25 \times 10^{6} \mathrm{kgm}^{-2} \mathrm{~s}^{-1} .
\end{aligned}
$$




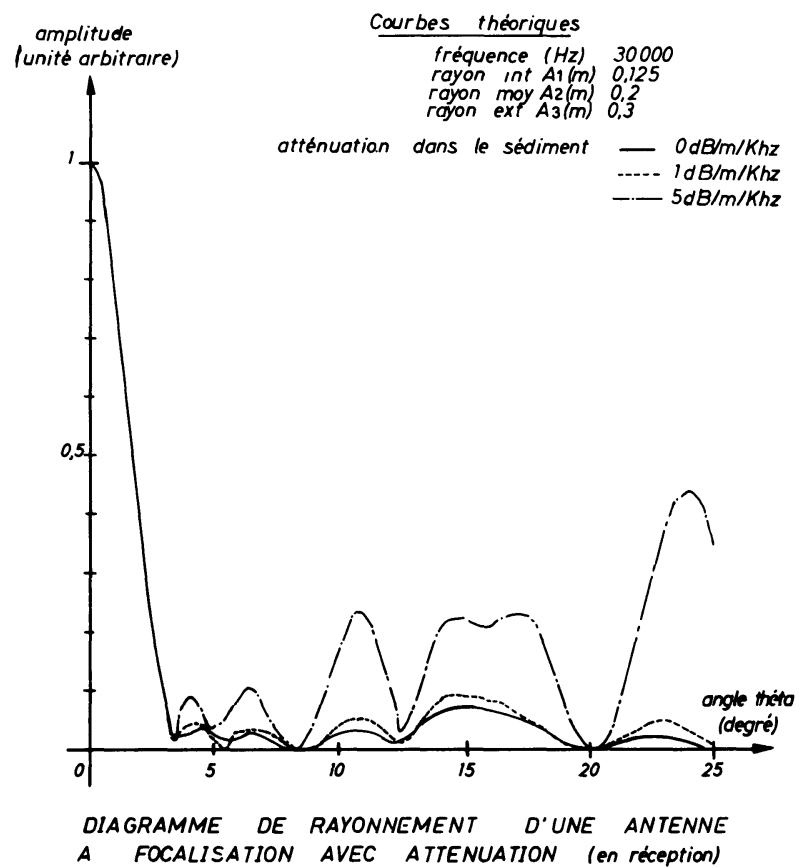

Fig. 5. - Diagramme de rayonnement (en réception) en présence d'une interface eau-sédiment.

[Response pattern (reception) in view of substract.]
Elles concernent le lobe de rayonnement en réception.

On notera des lobes secondaires avec l'atténuation dans le sédiment.

\section{Conclusion}

La hauteur de l'antenne au-dessus de l'interface doit être telle que les effets dus à la réflexion des ondes à l'interface soient négligeables (ou compensables). L'angle d'incidence des vibrations générées par la couronne extérieure et concernant le point de focalisation ne doit pas dépasser une trentaine de degrés pour un fonctionnement correct. $R_{i}\left(\rho, \theta_{i}\right)$ atteint l'unité lorsque $\rho$ et $\theta_{i}$ varient respectivement $2000 \mathrm{kgm}^{-3}$ et 45 à $50^{\circ}$. L'atténuation joue également un grand rôle : croissant avec la longueur des trajets acoustiques, les trajets les plus longs ne doivent pas présenter une grande différence avec les plus courts. Il y a donc lieu de choisir un compromis entre la perte dans le sédiment et la finesse de la tâche. De grands trajets facilitent l'emploi d'un modèle simple, mais ils sont limités par $\delta$ en regard de la fréquence des ondes utilisées.

\section{Bibliographie}

[1] Attenborough, K., Influence of microstructure on vicous attenuation acoustics and the sea end. (Bath University Press. Buth University Claverton Down. Bath. UK.) 1983.

[2] Bresson, J., Acquisition automatique et traitement de données appliquées à la caractérisation in situ des sédiments marins. Thèse Doct. Ing., Université de Montpellier, octobre 1979.

[3] Bresson, J., Longuemard, J. P., Mesure du coefficient à proximité du fond marin à des fréquences comprises entre 20 et $100 \mathrm{kHz}$. Applications à la détermination de certaines qualités géotechniques des sédiments du plateau continental français. Rev. Phys. Appl. 14 (1979).

[4] McDaniel, Stand, J. H., Semi-empirical sea bed models based on the Biot theory. Acoustics and the sea Bed. (Bath University Press Bath University Claverton Down Bath., U.K.) 1983.

[5] Frohly, J., Etude de la propagation des ondes ultrasonores dans les milieux hétérogènes et application de l'imagerie en mode $B$ au contrôle non destructif. Thèse d'Etat présentée à l'Université de Valenciennes, 1983.

[6] Hamilton, E. L., Sound velocity density relations in sea floor sediments and rocks. J. Acoust. Soc. Am. 63, 2 (1974).
[7] Hamilton, E. L., Prediction of deep sea sediment properties state of the art. Deep sea sediments (Plenum Press) 1973.

[8] Longuemard, J. P., Mesure in situ de l'amplitude des ondes réfléchies ou rétrodiffusées par les fonds marins. Application à la détermination in situ des modes granulométriques. Rev. Phys. Appl. 13 (1978).

[9] Moussiessie, J., Contribution à l'étude des relations entre l'acoustique et les qualités géotechniques des sédiments marins. Thèse $3 \mathrm{e}$ cycle. Université Paul Sabatier, Toulouse, soutenue à Perpignan, février 1984.

[10] Shibata, S., Tushio, K., Matsumgto, S., Yamaga, J., Coaxial circular spherical array for ultrasonic imaging. J. Acoustical Society America 62, $\mathrm{n}^{\circ} 4$ (1977).

[11] Shibata, S., Koda, T., Yamaga, J., $C$-mode ultrasonic imaging by an electronically scanned coaxial circular spherical receiving array. Ultrasonics, 1978.

[12] Stoll, R. P., Acoustic waves in satured sediments. Physics of sound in marine sediments. Marine Science (Plenum Press, New York) 1974.

[13] TAYLOR-SMITH, D., Acoustic and mechanical loading marin sediments. Marine Science (Plenum Press, New York) 1974. 